\title{
Tecnologías de Información y Rentabilidad de las Pymes del Sector Textil*
}

\author{
Leal Morantes, Miraidy Elena**
}

\section{Resumen}

El presente estudio tiene como objetivo analizar la relación entre el uso de las tecnologías de información y la rentabilidad de las pymes del sector textil ubicadas en el Municipio Maracaibo del Estado Zulia. La investigación se desarrolló a través de un estudio correlacional; para la recolección de datos se aplicó un cuestionario semiestructurado, bajo la modalidad de entrevista, a propietarios, presidentes, gerentes y administradores de 16 empresas. Los resultados evidencian que a pesar de los beneficios que brindan las tecnologías de información en la gestión empresarial, su uso, en el corto plazo, no contribuye al incremento de la rentabilidad en el sector estudiado; asimismo, factores como dificultad para acceder a mano de obra calificada, repuestos y soporte técnico adecuado, además de los altos costos de adquisición, limitan su uso. Se concluye que el incremento de la rentabilidad pudiera estar asociado a otros factores.

Palabras clave: Pequeñas y medianas empresas, rentabilidad, sector textil, tecnologías de información.

\section{Information Technologies and the Profitability of SAMEs in the Textile Sector}

\begin{abstract}
The objective of this study is to analyze the relationship between the use of information technologies and the profitability of textile sector SAMEs located in the Maracaibo Municipality of the State of Zulia. The research developed through a correlational study. To collect data, a semi-structured questionnaire was applied in the interview mode, to owners, presidents, managers and administra-
\end{abstract}

Recibido: 23-10-08. Aceptado: 15-09-09

* Avance del Programa Sistemas de Información Gerencial, adscrito al Centro de Estudios de la Empresa de la Facultad de Ciencias Económicas y Sociales de la Universidad del Zulia y financiado por el Consejo de Desarrollo Científico y Humanístico (CONDES).

** Licenciada en Contaduría Pública, Magíster en Gerencia de Empresas, Docente de la Escuela de Administración y Contaduría Pública de la Facultad de Ciencias Económicas y Sociales de la Universidad del Zulia, Venezuela. E-mail: mylealm@yahoo.es. Telf. (58) 0261-7596507. 
tors from 16 companies. Results show that despite the benefits offered by information technologies for business management, in the short term, their use does not contribute to a profitability increase in the sector studied; likewise, factors such as difficulty in obtaining qualified workers, replacement parts and technical support, as well as high acquisition costs, limit their use. Conclusions were that a profitability increase could be associated with other factors.

Key words: Small and medium enterprises, profitability, textile sector, information technologies.

\section{Introducción}

Actualmente, en el complejo mundo empresarial, se producen cambios vertiginosos de diversa índole que afectan a las organizaciones, por lo cual, para llevar a cabo una adecuada gestión éstas deben estar preparadas para enfrentar con éxito los desafíos del entorno.

Uno de los sectores económicos que se ha visto más afectado por esta situación es el de las pequeñas y medianas empresas (pymes). A pesar de ser un importante agente dinamizador de la economía, muchas de ellas se encuentran rezagadas tecnológicamente, por no contar con los recursos financieros necesarios para acceder a estas herramientas empresariales, lo cual dificulta su gestión empresarial y la posibilidad de competir exitosamente en el mercado.

Señalan Llisterri y Angelelli (2002: 14) que "la globalización está revolucionando los sistemas de producción y las estructuras industriales. En los nuevos esquemas de competencia, la calidad, el diseño, la atención a clientes y subcontratantes, la rapidez de los plazos de entrega y, sobre todo, la capacidad de innovación de las empresas, son los factores más importantes para crecer y consolidar la participación en los mercados internos y externos". Adicionalmente, se requiere que éstas sean productivas para obtener posiciones competitivas en el mercado.

En este sentido, el gobierno nacional ha diseñado e implementado una serie de políticas, programas e incentivos fiscales, dirigidos a la recuperación y fortalecimiento de este importante sector productivo del país. Dentro de las acciones propuestas se encuentra el desarrollo de políticas para elevar la productividad, la competitividad y la innovación, mediante el desarrollo de planes específicos para el mejoramiento de la productividad, que permita elevar la calidad de la producción industrial, reducir costos unitarios y masificar la producción, para hacerla más competitiva nacional e internacionalmente (Ministerio de Ciencia y Tecnología, 2004).

Debido a la importancia y participación que tienen las pymes en la economía venezolana y la relevancia que han adquirido los sistemas y tecnologías de información en la dinámica organizacional, en el presente estudio se analiza el uso de estas tecnologías y sus efectos en la rentabilidad empresarial, mediante la determinación de coeficientes de correlación, considerando las variables: a) inversiones tecnológicas, b) capacidad utilizada y c) años de uso de los equipos. Asimismo, se identifican las principales limitaciones que enfrentan las pymes para su 
Tecnologías de Información y Rentabilidad de las Pymes del Sector Textil Leal Morantes, Miraidy Elena

uso, tomando como referencia empírica 16 empresas, inscritas en el (SAMAT) ${ }^{1}$, pertenecientes al sector textil, ubicadas en el Municipio Maracaibo, Estado Zulia.

Para la selección de la muestra, se tomaron como criterios de clasificación que las pymes se dedicaran a la confección de prendas de vestir, con tres o más años en el mercado y con actividad económica durante el año 2007.

\section{Pymes, Tecnologías de Información e Innovación}

Las Tecnologías de Información (TI) consisten en el "conjunto convergente de tecnologías de la microelectrónica, la informática, las telecomunicaciones/televisión/radio y la optoelectrónica" (Castells, 1999: 56). Estas tecnologías han avanzado rápidamente, produciendo cambios en la economía mundial y modificando las formas de trabajar, ya que han permitido la automatización de los procesos y facilitado la innovación.

Estas situaciones experimentadas en el mercado mundial demandan cambios profundos en los estilos gerenciales. Cualquier organización, para sobrevivir y mantenerse, requiere de una adecuada y eficiente gerencia que permita alcanzar las metas y objetivos propuestos, de acuerdo a las oportunidades y limitaciones del medio en el que se encuentre inmersa.

La clave para su éxito radicaría en su capacidad para competir en un mercado globalizado, aprovechar al máximo sus fortalezas y ampliar sus objetivos de producción y rendimiento; para ello, se requiere que éstas sean productivas. Al respecto, plantean Mercado et al. (1998: 22) que cualquier empresa que desee sobrevivir a los cambios económicos que se producen actualmente, debe tener como uno de sus atributos la productividad, entendiéndose ésta como "...la relación entre los productos o servicios generados por un sistema... y los recursos utilizados para hacerlo".

Uno de los sectores más afectados por estos procesos es el de la pequeña y mediana empresa, el cual constituye un importante elemento dinamizador de la economía, como fuente generadora de empleo y por contribuir al producto interno bruto del país. En efecto, según cifras aportadas por el Consejo Nacional de Industrias (CONINDUSTRIA), para el año 2005 , el $91 \%$ de las empresas venezolanas eran pequeñas y medianas (aproximadamente 6.200 entidades), con una participación del $41 \%$ del empleo en el país $^{2}$. Es por ello que los gobiernos han

1 El Servicio Autónomo Municipal de Administración Tributaria (SAMAT), creado mediante ordenanza el 16 de Octubre de 2000 "...es el organismo encargado de administrar, fiscalizar, recaudar y controlar, aplicar sanciones y resguardar el sistema de los ingresos tributarios del Municipio Maracaibo y los impuestos nacionales que le sean delegados, con excepción de los relativos a las tasas atribuidas a otros organismos municipales" (www.samat.gob.ve).

2 Estas empresas se concentran principalmente en los Estados Miranda, Carabobo, Aragua y Zulia y los sectores en los cuales tienen mayor presencia son: confección, productos médicos quirúrgicos, artes gráficas y alimentos (www.mipunto.com, 2005). 
implementado una serie de acciones orientadas a dar apoyo a este sector productivo.

Dentro de este contexto, el Estado Venezolano ha diseñado una serie de políticas y programas destinados a brindar apoyo en materia de financiamiento, asistencia técnica, capacitación, desarrollo tecnológico, entre otros; por lo que ha creado una serie de disposiciones legales que constituyen el marco jurídico que regula el diseño y ejecución de dichas políticas, así como una serie de incentivos fiscales dirigidos a la recuperación y fortalecimiento de este sector productivo del país. Sin embargo, muchas de ellas presentan una serie de limitaciones que han dificultado su gestión empresarial y eficiencia operativa.

Tomando como referencia empírica algunos estudios efectuados a pymes de la región zuliana ${ }^{3}$, se determinó que entre los principales problemas que las caracterizan destacan los relacionados con el acceso y uso de las Tecnologías de Información.

Es indudable el impacto que ha producido el uso de estas tecnologías, no sólo en el ámbito empresarial, sino en la economía en general, incidiendo positivamente en los índices de productividad. Plantean Mercado et al. (1998) que la productividad puede considerarse como una medida de la eficiencia de la gestión empresarial, al evaluar la manera cómo se utilizan los recursos para la producción de bienes o servicios; asimismo, al ser productivas, las empresas pueden aumentar su competitividad, reducir sus costos e incrementar su rentabilidad, lo cual también ayudaría a la economía nacional, al garantizar fuentes de empleo.

Muchas organizaciones que han logrado el éxito empresarial se han apoyado en la aplicación de las TI en la búsqueda y consolidación de nuevos mercados para sus productos y la obtención de ventajas competitivas. Algunos estudios efectuados en décadas pasadas, entre los que destacan los realizados por Eric Brynjolfsson (1998), investigador del Instituto Tecnológico de Massachussets (Massachussets Institute of Technology - MIT-) y Harvard Business School (2005), evidencian una correlación positiva entre tecnologías de información y productividad.

Con base en estos resultados y los planteamientos de autores como Mercado et al. (1998), se podría suponer que las TI también tienen un impacto positivo en la rentabilidad empresarial y que a mayor uso de éstas aumentaría el retorno de la inversión, por lo cual se debería impulsar el uso masivo para una mejor gestión operativa. Sin embargo, no todas las empresas han aprovechado las ventajas que ofrecen estas nuevas tecnologías, principalmente el sector de las pequeñas y medianas empresas venezolanas.

El gobierno nacional, dentro de su plan de desarrollo económico y social, tiene como política en materia de TI el desa-

3 Uno de esos estudios fue llevado a cabo durante los años 2004-2005 por el Centro de Estudios de la Empresa de La Universidad del Zulia, en convenio con el FONACIT como parte del Programa de Formación de Emprendedores, Empresarios y Cooperativistas. 
Tecnologías de Información y Rentabilidad de las Pymes del Sector Textil Leal Morantes, Miraidy Elena

rrollo y uso intensivo de éstas en los distintos ámbitos de la sociedad. Con relación al sector productivo, están orientadas hacia el aumento de las capacidades en TI, siendo uno de los proyectos de acción la masificación del uso de herramientas TI en las pymes, microempresas, cooperativas y afines.

La implementación de estas políticas buscan, entre otros resultados, "Conformar capacidades de innovación productiva dentro de los pequeños productores y empresarios, para que con apoyo de las herramientas TIC, éstos puedan incrementar su productividad, y contribuyan con la generación de empleo y el desarrollo endógeno" (Ministerio de Ciencia y Tecnología, 2004).

A pesar de las políticas estatales orientadas a dar apoyo a este sector productivo, los niveles de utilización de tecnologías de información son pequeños, pudiendo incidir varios factores: restricciones financieras, resistencia de los empresarios a su utilización, relación costos-beneficios. Bajo este contexto, en la presente investigación se analiza la relación entre el uso de las tecnologías de información y la rentabilidad empresarial de las pymes del sector textil, ubicadas en el Municipio Maracaibo, Estado Zulia, con el fin de comprobar si su uso supone una mayor rentabilidad.

\section{Tecnologías de Información y Rentabilidad Empresarial}

Una de las condiciones para la supervivencia y crecimiento de cualquier organización consiste en adaptarse al entorno cambiante, así como incrementar su patrimonio, para lo cual se deben tomar decisiones cuyo rendimiento permita aumentar el valor de la empresa y el correspondiente a sus accionistas.

Establece Ortiz (1994: 365) que el objetivo final de cualquier organización productiva consiste en maximizar el rendimiento de la inversión mediante las utilidades y el crecimiento sostenido del capital, para lo cual deben cubrirse las siguientes 4 fases:

1. Establecer el monto de recursos que permitan el funcionamiento normal de la empresa.

2. Definir la combinación de financiamiento que garantice la operación, según la estimación de sus necesidades y la minimización del costo de cada fuente de financiamiento.

3. Orientar apropiadamente los recursos captados, considerando que cada fuente de financiamiento debería atender un renglón específico de la inversión y procurando evitar la subestimación de necesidades de capital o la tenencia de fondos ociosos no exigidos por las labores cotidianas de administración, producción, aprovisionamiento y comercialización.

4. Implantar mecanismos tendientes al máximo empleo de la capacidad instalada.

Una forma de evaluar los beneficios obtenidos por la empresa es a través de los índices de rentabilidad; para calcularlos, se debe tomar como base los resultados obtenidos (ganancia), pudiéndose determinar con relación a las ventas, los activos, al capital o al valor accionario (Gitman, 1997). En este sentido, la gestión gerencial debe concentrar sus esfuerzos en la obtención de utilidades, ya que con éstas las empresas atienden dos objetivos primordiales: la capitaliza- 
ción y el reconocimiento de dividendos o retribución del esfuerzo del inversionista (Ortiz, 1994: 370).

El aumento de las utilidades puede obtenerse mediante dos vías: por el incremento de los ingresos o por la reducción de los costos (Gitman, 1997), por lo tanto, un factor que incide en el aumento de la rentabilidad es el desarrollo tecnológico.

Señala Ortiz que (1994: 73) "por el impacto sobre las calidades y los costos de producción, es claro que las firmas actualizadas en materia tecnológica tienen amplias oportunidades de conquistar clientes, desplazar competidores, liderar el desarrollo de productos o captar mercados no cautivos", expresando, además, que la disponibilidad de recursos y procesos tecnológicos desarrollados origina ventajas competitivas y mayores utilidades, ya que se incrementa la productividad de los factores productivos, disminuye el desperdicio y la ociosidad, se optimiza el empleo del factor humano, ocasionando la reducción de costos, y por ende, ventajas competitivas en precios. Asimismo, autores como Gómez y Suárez (2004) plantean que la aplicación de las TICs en las pymes puede servir como soporte para la innovación de sus procesos, mediante la aplicación de diferentes herramientas.

Por el contrario, el atraso tecnológico produce desplazamiento del mercado, debido a calidades superiores de la competencia, menores niveles de productividad, originando subempleo de la capacidad instalada, incremento de los costos fijos y totales unitarios, declive de márgenes de utilidad y retroceso de índices de rentabilidad (Ortiz, 1994).

Son notables los beneficios que ofrece el uso de las TI, sin embargo, éstas no han sido aprovechadas por todas las empresas venezolanas, especialmente las pequeñas y medianas, incidiendo varios factores, básicamente de tipo financiero y cultural, lo cual ha propiciado dificultades en su gestión operativa.

\section{Tecnologías de Información en las Pymes del Sector Textil de Maracaibo}

Para efectuar el estudio, la muestra, constituida por 16 pymes, fue agrupada de acuerdo con el uso o no de las tecnologías de información en la gestión empresarial (Ver Tabla 1). Los principales productos que fabrican las empresas analizadas corresponden a tres categorías: uniformes y bragas (9 empresas; $56 \%$ ), franelas, camisas y chemises (4 empresas; $25 \%$ ), vestidos (3 empresas; 19\%). (Ver Tabla 2).

Tomando como referencia el criterio de clasificación mencionado, el $81 \%$ de las empresas estudiadas (13 empresas) utilizan las tecnologías de información: fabricación de uniformes y bragas (56\%, 9 empresas) y fabricación de franelas, camisas y chemises (25\%, 4 empresas) (Ver Tabla 2).

De las 13 empresas que utilizan tecnologías de información, sólo 9 (56\%) las aplican en su proceso productivo: fabricación de uniformes y bragas (37\%, 6 empresas) y fabricación de franelas, camisas y chemises (19\%, 3 empresas); las otras 4 empresas (25\%) las utilizan en otras áreas diferentes a producción: fabricación de uniformes y bragas (19\%, 3 empresas) y confección de franelas, camisas y chemises (6\%, 1 empresa). (Ver Tablas 1 y 3 ). 
Tecnologías de Información y Rentabilidad de las Pymes del Sector Textil Leal Morantes, Miraidy Elena

Tabla 1

Composición de la muestra por uso de tecnologías de información

\begin{tabular}{lcccccc}
\hline $\begin{array}{c}\text { Tecnologías } \\
\text { de Información }\end{array}$ & Total & \multicolumn{2}{c}{$\begin{array}{c}\text { Uso TI en el Proceso } \\
\text { Productivo }\end{array}$} & $\begin{array}{c}\text { No Uso TI en el } \\
\text { Proceso Productivo }\end{array}$ \\
\cline { 2 - 7 } & $\begin{array}{c}\text { No. de } \\
\text { Empresas }\end{array}$ & $\%$ & $\begin{array}{c}\text { No. de } \\
\text { Empresas }\end{array}$ & $\begin{array}{c}\text { No. de } \\
\text { Empresas }\end{array}$ & $\%$ \\
\hline Uso TI & 13 & $81 \%$ & 9 & $56 \%$ & 4 & $25 \%$ \\
No Uso TI & 3 & $19 \%$ & 0 & $0 \%$ & 3 & $19 \%$ \\
Total & 16 & $100 \%$ & 9 & $56 \%$ & 7 & $\mathbf{7 4 \%}$ \\
\hline
\end{tabular}

Fuente: Leal (2008).

Tabla 2

Composición de la muestra por principales productos fabricados y uso de tecnologías de información

\begin{tabular}{lcccccc}
\hline \multicolumn{1}{c}{$\begin{array}{c}\text { Principales } \\
\text { Productos }\end{array}$} & $\begin{array}{c}\text { Total } \\
\text { No. de } \\
\text { Empresas }\end{array}$ & $\begin{array}{c}\text { Uso TI } \\
\text { No. de } \\
\text { Empresas }\end{array}$ & $\begin{array}{c}\text { No Uso deTI } \\
\text { No. de } \\
\text { Empresas }\end{array}$ & $\%$ \\
\hline $\begin{array}{l}\text { Uniformes y Bragas } \\
\text { Franelas, Camisas }\end{array}$ & 9 & $56 \%$ & 9 & $56 \%$ & 0 & $0 \%$ \\
$\begin{array}{l}\text { y Chemises } \\
\text { Vestidos }\end{array}$ & 4 & $25 \%$ & 4 & $25 \%$ & 0 & $0 \%$ \\
Total & 16 & $19 \%$ & 0 & $0 \%$ & 3 & $19 \%$ \\
\hline
\end{tabular}

Fuente: Leal (2008).

El $19 \%$ de las empresas estudiadas (3), dedicadas a la confección de vestidos, no utilizan tecnologías de información en ninguna de sus áreas (Ver Tablas 1 y 2$)$.

\section{Rentabilidad en las Pymes del Sector Textil de Maracaibo}

La rentabilidad, definida como el rendimiento o beneficio obtenido por los recursos invertidos, está influenciada por dos factores fundamentales: los ingresos y costos de la organización. Señala Gittman (1997) que las empresas más rentables suelen ser aquellas con la ma- yor participación de mercado para sus productos; asimismo, Ortiz (1994) establece que las empresas con recursos y procesos tecnológicos desarrollados obtienen mayores utilidades, debido al incremento de la productividad de los factores productivos y a la disminución de costos.

De acuerdo con estos planteamientos, es de suponer que aquellas empresas con mayor nivel de ingresos e inversión tecnológica, deberían tener mayores niveles de rentabilidad. Sin embargo, los datos obtenidos en las empresas analizadas contradicen esta afirmación (Ver Tabla 4). 
Tabla 3

Composición de la muestra por principales productos fabricados y uso de tecnologías de información en el proceso productivo

\begin{tabular}{lcccccc}
\hline \multicolumn{1}{c}{$\begin{array}{c}\text { Principales } \\
\text { Productos }\end{array}$} & \multicolumn{1}{c}{ Total } & \multicolumn{2}{c}{$\begin{array}{c}\text { Uso TI en el Proceso } \\
\text { Productivo }\end{array}$} & \multicolumn{2}{c}{$\begin{array}{c}\text { No Uso TI en el Proceso } \\
\text { Productivo }\end{array}$} \\
\cline { 2 - 7 } & $\begin{array}{c}\text { No. de } \\
\text { Empresas }\end{array}$ & $\begin{array}{c}\text { No. de } \\
\text { Empresas }\end{array}$ & $\begin{array}{c}\text { No. de } \\
\text { Empresas }\end{array}$ & $\%$ \\
\hline $\begin{array}{l}\text { Uniformes } \\
\text { y Bragas }\end{array}$ & 9 & $56 \%$ & 6 & $37 \%$ & 3 & $19 \%$ \\
$\begin{array}{l}\text { Franelas, } \\
\text { Camisas y }\end{array}$ & 4 & $25 \%$ & 3 & $19 \%$ & 1 & $6 \%$ \\
Chemises & & & & & & \\
Vestidos & 0 & $0 \%$ & 0 & $0 \%$ & 0 & $0 \%$ \\
Total & 13 & $81 \%$ & 9 & $56 \%$ & 4 & $\mathbf{2 5 \%}$ \\
\hline
\end{tabular}

Fuente: Leal (2008).

Con base en información suministrada por las personas entrevistadas, la rentabilidad promedio del sector textil se ubica entre un 25\%-30\%. Para el año 2007 , las dos empresas más rentables de la muestra tienen un nivel de rentabilidad superior al promedio del mercado y las tres menos rentables tienen un nivel inferior al del mercado ${ }^{4}$ (Ver Tabla 5). El resto de las empresas se encuentran en el rango $25 \%-30 \%$.

La empresa más rentable de la muestra lleva a cabo un proceso productivo caracterizado por un uso intensivo de la mano de obra, con máquinas y equipos industriales que no están avanzados tecnológicamente, por ejemplo, no tienen máquinas bordadoras. Cuando un cliente solicita la confección de uniformes o bragas que requieren bordado, este servicio es subcontratado.
Para la empresa es más favorable subcontratar este servicio porque la adquisición de este tipo de maquinarias (importadas) requiere desembolsos de recursos considerables, si la empresa no posee los recursos suficientes debe recurrir al financiamiento externo (generalmente de la banca privada), con la consiguiente carga financiera (intereses); asimismo, cuando se le dificulta la consecución de divisas debe recurrir al mercado paralelo, incrementándose los costos de adquisición.

Por otra parte, para poder recuperar la inversión en un tiempo prudencial sería necesario emplear al máximo la capacidad productiva, ameritando, en algunos casos, trabajar turnos adicionales, lo cual sería innecesario tomando en consideración la demanda. Asimismo, existen otros factores que limitan el uso de estos recursos tecnológicos:

$4 \quad$ El índice de rentabilidad utilizado para esta investigación es el margen neto de utilidades (utilidad neta/ventas), utilizado como referencia en el sector estudiado. 
Tecnologías de Información y Rentabilidad de las Pymes del Sector Textil Leal Morantes, Miraidy Elena

Tabla 4

Niveles de Rentabilidad, Ingresos e Inversiones Tecnológicas de las Pymes Año 2007

\begin{tabular}{lccc}
\hline Empresa $^{5}$ & Rentabilidad (\%) & Ingresos (Bs. F.) & $\begin{array}{c}\text { Inversión Tecnológica } \\
\text { (Bs. F) }\end{array}$ \\
\hline Empresa 1 & 20 & 770.000 & 50.000 \\
Empresa 2 & 35 & 1.500 .000 & 45.000 \\
Empresa 3 & 30 & 350.000 & 30.000 \\
Empresa 4 & 20 & 15.000 & 10.300 \\
Empresa 5 & 6 & 652.425 & 8.000 \\
Empresa 6 & 35 & 390.000 & 6.000 \\
Empresa 7 & 30 & 400.000 & 0 \\
Empresa 8 & 28 & 24.000 & 0 \\
Empresa 9 & 27 & 18.000 & 0 \\
Empresa 10 & 26 & 30.000 & 0 \\
Empresa 11 & No Respondió & No Respondió & 0 \\
Empresa 12 & No Respondió & No Respondió & 0 \\
Empresa 13 & No Respondió & No Respondió & 0 \\
Empresa 14 & No Respondió & No Respondió & 0 \\
Empresa 15 & No Respondió & No Respondió & 0 \\
Empresa 16 & No Respondió & No Respondió & 0 \\
\hline Fun : & & &
\end{tabular}

Fuente: Leal (2008).

Dificultad para la consecución de repuestos y soporte técnico adecuado. Una de las empresas de la muestra, para el momento de la entrevista, tenía dos meses sin utilizar una de las máquinas porque no se conseguía una pieza que estaba dañada, lo cual trae como consecuencia, la subutilización de la capacidad instalada e incremento de los costos.

Dificultad para la consecución de mano de obra calificada, no sólo para el manejo adecuado de estas maquinarias, sino también personal con experiencia en el área de corte y costura. Esta situación, aunado a la dificultad para la consecución de insumos constituyen los principales problemas señalados en el proceso de producción (Ver Tabla 6).

Demanda reducida, debido a que la mayoría de las empresas analizadas sólo tienen presencia en el mercado regional (Ver Tabla 7), esta situación limita el interés de invertir con el fin de ampliar la capacidad instalada, centrando el interés en

5 Por solicitud de los propietarios, se omite el nombre de las empresas. 
Tabla 5

Empresas Más Rentables y Menos Rentables Año 2007

\begin{tabular}{lccc}
\hline \multicolumn{1}{c}{ Empresa } & Producto Principal & Rentabilidad & $\begin{array}{c}\text { Uso TI en el Proceso } \\
\text { Productivo }\end{array}$ \\
\hline Empresa 6 & Uniformes y bragas & $35 \%$ & No \\
Empresa 2 & Chemises y franelas & $35 \%$ & Sí \\
Empresa 4 & Vestidos & $20 \%$ & No \\
Empresa 1 & Chemises & $20 \%$ & Sí \\
Empresa 5 & Uniformes & $6 \%$ & Sí
\end{tabular}

Tabla 6

Principales Problemas en el Área de Producción

\begin{tabular}{lcc}
\hline \multicolumn{1}{c}{$\begin{array}{c}\text { Principales } \\
\text { Problemas }\end{array}$} & $\begin{array}{c}\text { No. de } \\
\text { Empresas }\end{array}$ & $\%$ \\
\hline $\begin{array}{l}\text { Consecución de } \\
\text { Personal Capacitado }\end{array}$ & 8 & $50 \%$ \\
$\begin{array}{l}\text { Consecución de } \\
\text { Materiales }\end{array}$ & 8 & $50 \%$ \\
Total & 16 & $\mathbf{1 0 0 \%}$ \\
\hline
\end{tabular}

Fuente: Leal (2008).

la sustitución y mejora de equipos existentes (Ver Tabla 8). Cuando se producen pedidos excepcionales, que superan la capacidad instalada de la empresa, se recurre a la subcontratación de otros talleres dedicados a la confección de prendas de vestir; alegan los empresarios que es más económico esta subcontratación que la ampliación de la capacidad, debido al ahorro de algunos costos (por ejemplo, las cargas laborales que implicaría la contratación de nuevo personal, aunado a la dificultad para conseguirlo).

Con relación al nivel de ingresos, de las empresas más rentables y menos
Tabla 7 Oferta por Tipo de Mercado

\begin{tabular}{lcc}
\hline \multicolumn{1}{c}{ Mercado } & $\begin{array}{c}\text { No. de } \\
\text { Empresas }\end{array}$ & \multicolumn{1}{c}{$\%$} \\
\hline Regional & 11 & $69 \%$ \\
Regional y Nacional & 4 & $25 \%$ \\
Regional, Nacional & 1 & $6 \%$ \\
y Extranjero & & \\
Total & $\mathbf{1 6}$ & $\mathbf{1 0 0 \%}$ \\
\hline
\end{tabular}

Fuente: Leal (2008).

rentables del año 2007, las tres que utilizan las tecnologías de información en el proceso productivo son las que obtuvieron mayores ingresos durante el año 2007 (Ver Tablas 4 y 5), señalando los entrevistados como principales motivos la calidad de los productos, precios competitivos y tiempo de entrega. Sin embargo, sólo una tiene una rentabilidad superior al promedio del mercado, las otras dos empresas presentan niveles de rentabilidad inferiores.

La mayoría de los empresarios entrevistados consideran que el uso de tecnologías de información contribuye a aumentar la rentabilidad, mediante el incre- 
Tecnologías de Información y Rentabilidad de las Pymes del Sector Textil Leal Morantes, Miraidy Elena

Tabla 8

Finalidad de la Inversión Tecnológica Años 2005-2007

\begin{tabular}{lccc}
\hline & $\begin{array}{c}2007 \\
\text { No. de Empresas }\end{array}$ & $\begin{array}{c}\mathbf{2 0 0 6} \\
\text { No. de Empresas }\end{array}$ & $\begin{array}{c}\mathbf{2 0 0 5} \\
\text { No. de Empresas }\end{array}$ \\
\hline Sustitución de equipos & 3 & 2 & 2 \\
Mejoras en los equipos & 1 & 1 & 0 \\
$\begin{array}{l}\text { Ampliación de la capacidad } \\
\text { tecnológica }\end{array}$ & 2 & 2 & 1 \\
\hline Fuente: Leal (2008). & & &
\end{tabular}

Tabla 9

Información sobre Pymes

Año 2007

\begin{tabular}{lcccccc}
\hline Empresa & $\begin{array}{c}\text { Rentabilidad } \\
\text { (\%) }\end{array}$ & $\begin{array}{c}\text { Ingresos } \\
\text { (Bs. F.) }\end{array}$ & $\begin{array}{c}\text { Inversión } \\
\text { Tecnológica } \\
\text { (Bs. F) }\end{array}$ & $\begin{array}{c}\text { Capacidad } \\
\text { Utilizada } \\
\text { (\%) }\end{array}$ & $\begin{array}{c}\text { Años de } \\
\text { Uso } \\
\text { Equipos }\end{array}$ & $\begin{array}{c}\text { Principales } \\
\text { Productos }\end{array}$ \\
\hline Empresa 1 & 20 & 770.000 & 50.000 & $100 \%$ & 6 & Chemises \\
Empresa 2 & 35 & 1.500 .000 & 45.000 & $60 \%$ & 18 & Chemises \\
Empresa 3 & 30 & 350.000 & 30.000 & $85 \%$ & 6 & Uniformes \\
Empresa 4 & 20 & 15.000 & 10.300 & $100 \%$ & 2 & Vestidos \\
Empresa 5 & 6 & 652.425 & 8.000 & $90 \%$ & 3 & Uniformes \\
Empresa 6 & 35 & 390.000 & 6.000 & $80 \%$ & 3 & Uniformes \\
Empresa 7 & 30 & 400.000 & 0 & $100 \%$ & 3 & Chemises \\
Empresa 8 & 28 & 24.000 & 0 & $80 \%$ & 28 & Bragas \\
Empresa 9 & 27 & 18.000 & 0 & $100 \%$ & 3 & Vestidos \\
Empresa 10 & 26 & 30.000 & 0 & $100 \%$ & 16 & Vestidos \\
Empresa 11 & No respondió & No respondió & 0 & $70 \%$ & 6 & Uniformes \\
Empresa 12 & No respondió & No respondió & 0 & $100 \%$ & No respondió & Uniformes \\
Empresa 13 & No respondió & No respondió & 0 & $70 \%$ & No respondió & Bragas \\
Empresa 14 & No respondió & No respondió & 0 & $100 \%$ & No respondió & Bragas \\
Empresa 15 & No respondió & No respondió & 0 & $100 \%$ & No respondió & Uniformes \\
Empresa 16 & No respondió & No respondió & 0 & $90 \%$ & No respondió & Camisas \\
\hline Fun
\end{tabular}

Fuente: Leal (2008).

mento de ingresos o reducción de costos, sin embargo, esto no es respaldado por las inversiones realizadas en los últimos años; durante el año 2007, sólo 6 empre- sas invirtieron recursos en tecnología (Ver Tabla 4).

De acuerdo con el monto de la inversión, para el año 2007, de las empre- 
sas que ocupan los tres primeros lugares, 2 las han efectuado para ampliar la capacidad instalada, sin embargo, con excepción de la Empresa No. 2, no son las más rentables (Ver Tabla 4). La más rentable es la que ocupa el sexto lugar, la cual ha efectuado inversiones para mejorar los equipos existentes en el área de administración.

Con base en los resultados presentados, el uso de las tecnologías de información no incrementa la rentabilidad de las empresas del sector textil de Maracaibo para el período analizado, sin embargo, además del monto de las inversiones tecnológicas efectuadas en el año 2007, se consideraron otros parámetros, tales como la capacidad utilizada y los años de uso de los equipos (Ver Tabla 9), para determinar si existe correlación entre estas variables y la rentabilidad. Los resultados demuestran que la correlación es poco significativa (Ver Tabla 10), por lo que se presume que son otros los factores determinantes de la rentabilidad de este sector económico.

\section{Conclusiones}

Muchas organizaciones han logrado el éxito empresarial apoyadas en la aplicación de nuevas tecnologías de información, orientadas hacia la búsqueda y consolidación de nuevos mercados para sus productos y la obtención de ventajas competitivas.

Son indudables los beneficios que proporcionan las TI en la gestión empresarial, en efecto, los propietarios y gerentes consideran importante el uso de estas

Tabla 10

Coeficientes de Correlación

Año 2007

\begin{tabular}{cccccc}
\hline Empresa & $\begin{array}{c}\text { Rentabilidad } \\
(\mathbf{\%})\end{array}$ & $\begin{array}{c}\text { Ingresos } \\
\text { (Bs. F.) }\end{array}$ & $\begin{array}{c}\text { Inversión } \\
\text { Tecnológica } \\
\text { (Bs. F) }\end{array}$ & $\begin{array}{c}\text { Capacidad } \\
\text { Utilizada (\%) }\end{array}$ & $\begin{array}{c}\text { Años de } \\
\text { Uso } \\
\text { Equipos }\end{array}$ \\
\hline Empresa 1 & 20 & 770.000 & 50.000 & $100 \%$ & 6 \\
Empresa 2 & 35 & 1.500 .000 & 45.000 & $60 \%$ & 18 \\
Empresa 3 & 30 & 350.000 & 30.000 & $85 \%$ & 6 \\
Empresa 4 & 20 & 15.000 & 10.300 & $100 \%$ & 2 \\
Empresa 5 & 6 & 652.425 & 8.000 & $90 \%$ & 3 \\
Empresa 6 & 35 & 390.000 & 6.000 & $80 \%$ & 3 \\
Empresa 7 & 30 & 400.000 & 0 & $100 \%$ & 3 \\
Empresa 8 & 28 & 24.000 & 0 & $80 \%$ & 28 \\
Empresa 9 & 27 & 18.000 & 0 & $100 \%$ & 3 \\
Empresa 10 & 26 & 30.000 & 0 & $100 \%$ & 16 \\
Coeficiente & & & & & $\mathbf{0 , 3 0}$ \\
Correlación & & $\mathbf{0 , 1 0}$ & $\mathbf{0 , 0 7}$ & & \\
\hline
\end{tabular}

Fuente: Leal (2008). 
Tecnologías de Información y Rentabilidad de las Pymes del Sector Textil Leal Morantes, Miraidy Elena

tecnologías, sin embargo, en el estudio efectuado, sólo 9 (56\%) las aplican en su proceso productivo: fabricación de uniformes y bragas (37\%, 6 empresas) y fabricación de franelas, camisas y chemises (19\%, 3 empresas); 4 empresas $(25 \%)$ las utilizan en otras áreas diferentes a producción (ventas, administración), utilizando principalmente los programas a2, Valery y Saint.

El resto de las empresas estudiadas (3 empresas dedicadas a la confección de vestidos, 19\%) no utilizan tecnologías de información en ninguna de sus áreas. Esto se debe a que estas empresas tienen un proceso productivo donde las máquinas y equipos utilizados en la fabricación de prendas de vestir no son automatizados, lo cual requiere un uso intensivo del factor humano, además de otras causas, como la poca capacitación, altos costos o poca relevancia de su uso para la actividad empresarial.

Si bien el uso de tecnologías de información debería contribuir a la expansión y crecimiento empresarial, las pymes con mayor nivel de ingresos para el año 2007 utilizan las tecnologías de información en su proceso productivo, sin embargo, sólo una presenta un índice de rentabilidad superior al promedio del mercado, las otras dos empresas presentan niveles de rentabilidad inferiores. Igual sucede con aquellas empresas con mayores montos en inversiones tecnológicas, las que ocupan los tres primeros lugares, con excepción de una, no son las más rentables. La más rentable es la que ocupa el sexto lugar, cuyas inversiones se han efectuado para mejorar los equipos existentes en el área de administración.
A pesar de los beneficios que brindan las tecnologías de información en la gestión empresarial, demostrado por diversos estudios internacionales que confirman que su uso tiene efectos positivos en los niveles de productividad y rentabilidad, en el sector estudiado éstas no contribuyen al incremento de la rentabilidad (en el corto plazo); algunos factores como la dificultad para acceder a mano de obra calificada, repuestos y soporte técnico adecuado, además de los altos costos de adquisición, limitan su uso.

La empresa más rentable de la muestra lleva a cabo un proceso productivo caracterizado por un uso intensivo de la mano de obra, con máquinas y equipos industriales que no están avanzados tecnológicamente.

Con base en lo anterior, se puede presumir que la rentabilidad pudiera estar condicionada por otros factores, por lo que sería interesante identificar los factores determinantes de la rentabilidad en el sector textil de Maracaibo.

\section{Referencias Bibliográficas}

Brynjolfsson Erik y Hitt Lorin (1998). "Beyond the Productivity Paradox". Communications of ACM. Vol. 41, No. 8. pp. 49-55. Disponible en: http://cispom.boisestate.edu/cis320emaxson/prod982.pdf. Fecha de Consulta: 29/08/2007.

Castells, Manuel (1999). La Era de la Información. Economía, Sociedad y Cultura, La Sociedad Red. Vol. I, México, Siglo Veintiuno Editores, S.A.

Gitman, Lawrence (1997). Fundamentos de Administración Financiera. Traducción: Enrique Mercado y Juan Carlos Vega. Séptima Edición. Méxi- 
co. Oxford University Press Harla México, S.A. Pp. 1077.

Gómez, Álvaro y Suárez, Carlos (2004). Sistemas de Información. Herramientas prácticas para la Gestión Empresarial. México. Alfaomega Grupo Editor, S.A. Pp 201.

Harvard Business School (2005). Working Paper Series, No. 06-013. Why IT Matters in Midsized Firms. Fecha de Consulta: 29/08/2007.

Http://www.samat.gob.ve/index.php?option= com content\&task=view\&id=5\&ltemid=6. Fecha de Consulta: $01 / 05 /$ 2008.

Llisterri, Juan y Angelelli, Pablo (2002). Guía Operativa para Programas de Competitividad para la Pequeña y Mediana Empresa. Serie de Buenas Prácticas del Departamento de Desarrollo Sostenible No. MSM - 117. División de Micro, Pequeña y Mediana Empresa. Banco Interamericano de Desarrollo. Washington, D.C.
Mercado, Ernesto; Díaz, Esther y Flores, M. Diana (1998). Productividad. Base de la Competitividad. México. Editorial Limusa, S.A. de C.V. Grupo Noriega Editores.

Ministerio de Ciencia y Tecnología (2004). Informe de País. Objetivos de Desarrollo del Milenio para Venezuela. Estado de Situación y Marco de Política de las Tecnologías de Información y Comunicación en Venezuela. Http:// www.mct.gob.ve/uploads/biblio/Metas\%20del\%Mienio.pdf. Fecha de Consulta: 5/11/2005.

Mipunto.com (2005). 91\% de empresas venezolanas son pymes Disponible en: http://www.mipunto.com/punto_empresas/000/001/000/109.jsp. Fecha de Consulta: 27/08/2007.

Ortiz Gómez, Alberto (1994). Gerencia Financiera. Un Enfoque Estratégico. McGraw Hill Interamericana, S.A. Santafé de Bogotá, Colombia. 